EESTI NSV TEADUSTE AKADEEMIA TOIMETISED. 29. KOIDE FOOSIKA * MATEMAATIKA. 1980, NR. 4

ИЗВЕСТИЯ АКАДЕМИИ НАУК ЭСТОНСКОИ ССР. ТОМ 29 ФИЗИКА * МАТЕМАТИКА. 1980, № 4

\title{
ПРИМЕНЕНИЕ РАЗЛОЖЕНИЯ БРОМВИЧА В ЗАДАЧЕ КРУЧЕНИЯ МНОГОСЛОЙНОЙ СРЕДЫ
}

A. PURO. BROMWICHI JAOTUSE KASUTAMINE MITMEKIHILISE KESKKONNA POORLEMISE OLESANDES

A. PURO. THE APPLICATION OF THE BROMWICH EXPANSION METHOD IN SOLVING THE PROBLEM OF THE TWISTING OF LAYERED MEDIUM

(Представил Н. Алумяэ)

В пространстве трансформант Ганкеля $u(s, z), \sigma(s, z)$

$$
\begin{aligned}
& u_{\theta}(r, z)=\int_{0}^{\infty} s u(s, z) J_{1}(s, r) d s, \\
& \sigma_{\theta z}(r, z)=\int_{0}^{\infty} s \sigma(s, z) J_{1}(s, r) d s
\end{aligned}
$$

осесимметричная задача кручения сосредоточенным моментом вращения

$$
\begin{gathered}
2 \pi \int_{0}^{\infty} r^{2} \sigma_{\theta z}\left(r, z_{1}\right) d r=1, \quad \sigma_{\theta z}\left(r, z_{1}\right)=\delta(r) / 2 \pi r^{2}, \\
\sigma\left(s, z_{1}\right)=s / 4 \pi, \quad \delta(r)-\text { дельта функция, }
\end{gathered}
$$

приложенным к поверхности многослойного полупространства, сводится к решению системы уравнений $\left[{ }^{1,2}\right]$

$$
\frac{d}{d z}\left\|\begin{array}{c}
u \\
\sigma
\end{array}\right\|=\left\|\begin{array}{cc}
0 & \mu_{k}^{-1} \\
S^{2} \mu_{k} & 0
\end{array}\right\|\left\|\begin{array}{c}
u \\
\sigma
\end{array}\right\|
$$

где $\mu_{k}-$ коэффициент жесткости $k$-го слоя, $u_{\theta}-$ угловое смещение, $\sigma_{\theta z}$ - компонента тензора напряжений, ось $z$ совпадает с осью вращения и направлена к поверхности полупространства.

При $s=i \omega$ система уравнений (1) переходит в систему уравнений распространения акустических колебаний в многослойной среде относительно трансформант Фурье в случае нормального падения. Слои имеют единичную скорость звука и различные волновые сопротивления. Систему (1) можно записать и как уравнение второго порядка $u^{\prime \prime}-s^{2} u=0$ с надлежащими условиями сшивания на границе слоев. Учитывая взаимно-однозначное соответствие рассматриваемых динамической и статической задач, получим разложение Бромвича решения задачи кручения. 
Решение системы $B_{k}(s, z)$ в $k$-м слое

$$
B_{k}(s, z)=A\left(z-z_{k-1}\right) \prod_{n=0}^{k-2} A\left(z_{n+1}-z_{n}\right) B_{0}(s)
$$

выражается через матрицу $A$

$$
A\left(z-z_{k}\right)=\left\|\begin{array}{cr}
\operatorname{ch} s\left(z-z_{k}\right) & \left(\mu_{k} s\right)^{-1} \\
\mu_{k} s \operatorname{sh} s\left(z-z_{k}\right) & \operatorname{ch} s\left(z-z_{k}\right)
\end{array}\right\| .
$$

Угловое смещение $u_{k}(s, z)$ в $k$-м слое

$$
u_{k}(s, z)=a_{k} \mathrm{e}^{s z}+b_{k} \mathrm{e}^{-s z}
$$

можно выразить также, как и в динамическом случае $\left[{ }^{3}\right]$, через соответственно введенные коэффициенты отражения и прохождения

$$
\begin{array}{r}
c_{k}=\left(\mu_{k+1}-\mu_{k}\right) /\left(\mu_{k+1}+\mu_{k}\right), \quad t_{k}=2 \mu_{k+1} /\left(\mu_{k+1}+\mu_{k}\right), \\
\left\|\begin{array}{cc}
a_{k+1} \\
b_{k+1}
\end{array}\right\|=\frac{1}{t_{k}}\left\|\begin{array}{cc}
1 & c_{k} \mathrm{e}^{-2 s z_{k}} \\
c_{k} \mathrm{e}^{2 s z_{k}} & 1
\end{array}\right\|\left\|\begin{array}{l}
a_{k} \\
b_{k}
\end{array}\right\| .
\end{array}
$$

Так, в случае одного слоя, спаянного с полупространством, при $z=0$ решение в слое представим в виде разложения Бромвича

$$
u_{1}(s, z)=\frac{\mathrm{e}^{-s\left(z_{1}-z\right)}+c_{0} \mathrm{e}^{-s\left(z_{1}+z\right)}}{4 \pi \mu_{1}\left(1-c_{0} \mathrm{e}^{-2 s z_{1}}\right)}=\frac{1}{4 \pi \mu_{1}}\left[\mathrm{e}^{-s\left(z_{1}-z\right)}+c_{0} \mathrm{e}^{-s\left(z_{1}+z\right)}\right] \sum_{k=0}^{\infty}\left(c_{0} \mathrm{e}^{-2 s z_{1}}\right)^{k} .
$$

Выполняя обратное преобразование и учитывая, что

$$
\int_{0}^{\infty} s \mathrm{e}^{-s k} J_{1}(s r) d s=r /\left(r^{2}+k^{2}\right)^{3 / 2}=\varphi_{k}(r),
$$

получаем

$$
\begin{aligned}
u_{0}(r, z)= & \frac{1}{4 \pi \mu_{1}} \frac{\partial}{\partial r}\left[\sum_{k=0}^{\infty} \frac{c_{0}^{k}}{\sqrt{r^{2}+\left[(2 k+1) z_{1}-z\right]^{2}}}+\right. \\
& \left.+\frac{c_{0}^{k+1}}{\sqrt{r^{2}+\left[(2 k+1) z_{1}+z\right]^{2}}}\right] .
\end{aligned}
$$

Коэффициенты ряда можно вычислить как произведение коэффициентов, соответствующих кинематическому коду $\left[{ }^{4,5}\right]$, а знаменатель равен оптическому пути того же кода с учетом того, что угол падения равен углу отражения; при этом коэффициент. отражения от свободной поверхности равен единице.

Решение в полупространстве через коэффициенты отражения и прохождения

$$
u_{0}(s, z)=t_{0} \mathrm{e}^{s\left(z-z_{1}\right) /\left(4 \pi \mu_{1}\left(1-c_{0} \mathrm{e}^{-2 s z_{1}}\right)\right)}
$$

выражается в виде ряда

$$
u_{0}(r, z)=\frac{t_{0}}{4 \pi \mu_{1}} \frac{\partial}{\partial r} \sum_{k=0}^{\infty} \frac{c_{0}^{k}}{\sqrt{r^{2}+\left[(2 k+1) z_{1}-z\right]^{2}}} .
$$


При рассмотрении задач, содержащих три слоя (и более), можно применить технику расчета, разработанную в акустике $[4,5]$, причем ряды, получаемые таким образом, сходятся быстрее, чем в динамическом случае, так как они содержат множителем величину порядка $1 / k$.

При расчете смещения в $k$-м слое коэффициенты прохождения, входящие в формулу расчета коэффициентов ряда (обобщенные коэффициенты отражения и прохождения), принимаются равными единице. Коэффициенты $t_{i}$ входят множителем $\prod_{i=k_{0}}^{k} t_{1}$ в члены ряда, где $k_{0}-$ номер слоя, в котором находится источник напряжения. Так, в случае двух слоев, скрепленных с полупространством, и поверхности, загруженной сосредоточенным моментом, смещение в полупространстве

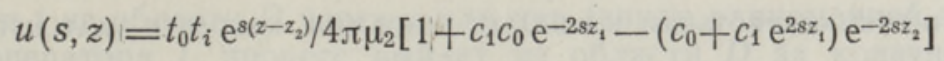

разлагается в ряд Бромвича и $t_{0} t_{1}$ входят множителем в этот ряд.

По коэффициентам разложения смещения в ряд Бромвича от действия сосредоточенного момента можно определить, в свою очередь, коэффициенты жесткости слоев. В частности, если слои имеют одинаковую толщину, равную 0,5 , то смещение на поверхности разлагается в ряд по функциям $\varphi_{k}(r)$, образующим полную систему функций с весом $r$ в пространстве $c(0, \infty)$. Полнота системы следует из полноты функций $\mathrm{e}^{-8 k}$ в $L_{2}[0, \infty)$. Алгоритм решения задачи при такой постановке такой же, как и в динамическом случае $\left[{ }^{3}\right]$. Некорректность обратной задачи проявляется в том, что система функций $\varphi_{k}(r)$ не является ортонормированной.

Рассмотренную технику разложения можно распространить на задачи сжатия многослойной ореды, причем использовать для расчетов на ЭВМ пропраммы, составленные для задач акустики.

Автор признателен Н. Векслеру за полезные обсуждения работы.

\section{ЛИТЕРАТУ РА}

1. Н а у мо в Ю. А., Ч и ст як В. И., Прикл. механика, XX, № 8, 40-50 (1979).

2. Н и киши н В. С., Шапи ро Г. С., Задачи теории упругости для многослойных сред, М., «Наука», 1973 , с. $39-42$.

3. W a r e, J. A., A k i, K., J. Acoust. Soc. Amer., 45, № 4, 911-921 (1969).

4. Пет р ашень Г. И., В а в илов а Т. И., В кн.: Вопросы динамической теории распространения сейсмических волн, 9, Л., Изд-во Ленингр. ун-та, 1968, c. $77-96$.

5. Cerveny̌, V., Mol otkov, I. A., Pšenčik, I., Ray method in seismology, Univerzita Karlova Praha, Praha, 1977, p. 39-41.

Калининградский технический институт рыбной промышленности и хозяйства
Поступила в редакцию $22 / \mathrm{V} 1980$ 